\title{
Penny-Packing and Two-Dimensional Codes
}

\author{
R. L. Graham and N. J. A. Sloane \\ Mathematical Sciences Research Center, AT\&T Bell Laboratories, \\ Murray Hill, NJ 07974, USA
}

\begin{abstract}
We consider the problem of packing $n$ equal circles (i.e., pennies) in the plane so as to minimize the second moment $U$ about their centroid. These packings are also minimal-energy two-dimensional codes. Adding one penny at a time according to the greedy algorithm produces a unique sequence of packings for the first 75 pennies, and appears to produce optimal packings for infinitely many values of $n$. Several other conjectures are proposed, and a table is given of the best packings known for $n \leq 500$. For large $n, U \sim \sqrt{3} n^{2} /(4 \pi)$.
\end{abstract}

\section{The Penny-Packing Problem}

We wish to find the tightest packing in the plane of $n$ equal circular disks, pennies for example, for $n=1,2, \ldots$ Groemer, Wegner, and others have considered minimizing the area of the convex hull of the pennies [8], [15], but for our applications it is more appropriate to minimize their second moment.

Suppose the pennies have diameter $d$, let $P_{1}, \ldots, P_{n}$ be their centers and $\tilde{P}=n^{-1} \sum P_{i}$ their centroid. Then the problem is to choose points $P_{1}, \ldots, P_{n}$ so as to satisfy

$$
\left\|P_{i}-P_{j}\right\| \geq d, \quad i, j=1, \ldots, n, i \neq j
$$

and so that the second moment

$$
U=\frac{1}{d^{2}} \sum_{i=1}^{n}\left\|P_{i}-\bar{P}\right\|^{2}
$$

is minimized, where \|\| is the Euclidean distance. Let $U(n)$ be the minimal value of $U$ (which can always be attained). A set of points $\mathscr{P}=\left\{P_{1}, \ldots, P_{n}\right\}$ satisfying (1) is called an $n$-point packing, and is optimal if it attains $U(n)$. 
The second moment $U$ is a reasonable parameter to minimize, for several reasons. For applications to signal design, when the points $P_{1}, \ldots, P_{n}$ form a two-dimensional code [3], [4], [7], $U$ measures the energy in the code. Figure 1(b), for example, shows what is conjectured to be the 16-point code with the smallest possible energy [4]. Furthermore, the results look tightly packed (Fig. 1(a)-(c)), and when applied to packings in higher dimensions this criterion avoids the "sausage catastrophe" [5].

Penny packings also have applications in cluster chemistry [9], [14], quantiz-

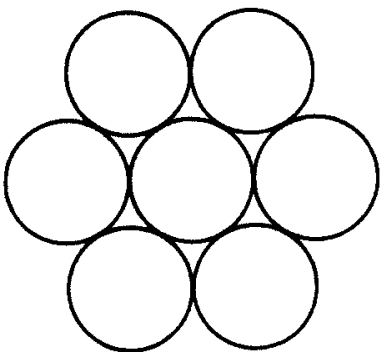

(a)

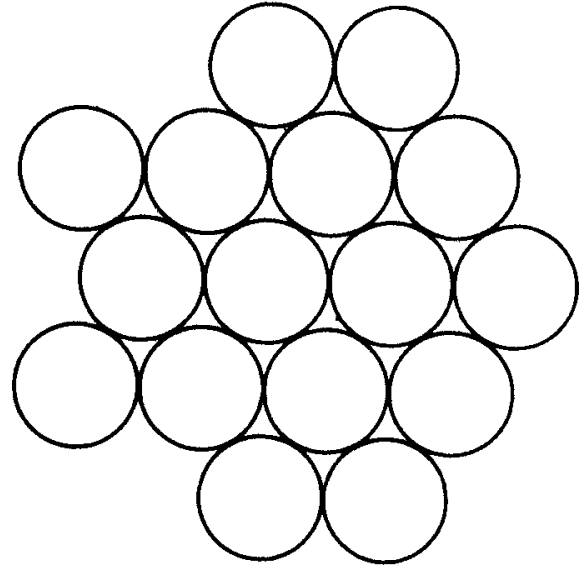

(b)

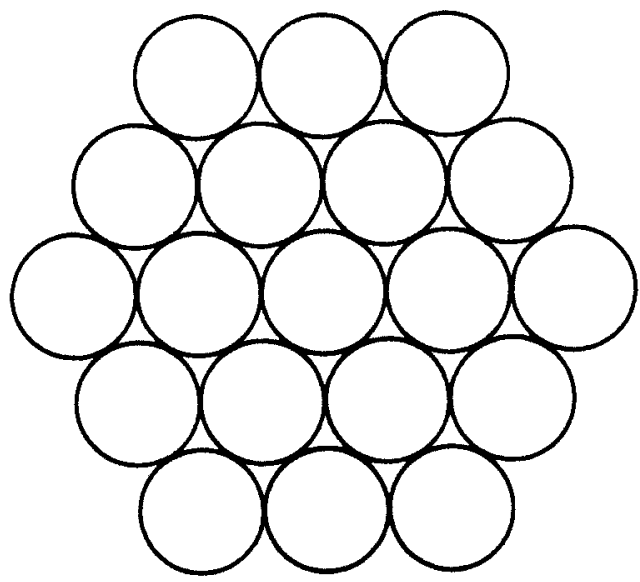

(c)

Fig. 1. Packings of 7,16 , and 19 pennies that are believed to have the smallest second moments. 
ation, and other areas [14]. Figure 1(c), for example, shows the 19 strands of wire in a cross-section of the cables used in the Brooklyn bridge [12]. The packing formed by pennies 1 to 37 in Fig. 4 occurs in the cross-section of an optical-fiber cable [11].

\section{The Hexagonal Lattice}

The familiar hexagonal lattice $A_{2}$ is spanned by $(1,0)$ and $\left(\frac{1}{2}, \sqrt{3} / 2\right)$. If we take $d=1$ then any subset of $A_{2}$ is a packing.

Conjecture 1. For $n \neq 4$ every optimal packing is (apart from a magnification, rotation and translation) a subset of $A_{2}$.

The case $n=4$ is exceptional, since all packings of the type shown in Fig. 2 have $U=2$ (as long as each penny touches its two neighbors), and in general these are not subsets of $A_{2}$. Our investigations suggest that $n=4$ is the only exception, although we cannot prove this.

Any point $P \in A_{2}$ can be written as

$$
r(1,0)+s\left(\frac{1}{2}, \frac{\sqrt{3}}{2}\right)=\left(r+\frac{s}{2}, \frac{s \sqrt{3}}{2}\right)
$$

for $r, s \in Z$; we call $\langle r, s\rangle$ oblique coordinates for $P$. There is a convenient formula for the second moment $U$ of a subset $\left\{P_{1}, \ldots, P_{n}\right\} \subseteq A_{2}$. Let $P_{i}$ have oblique

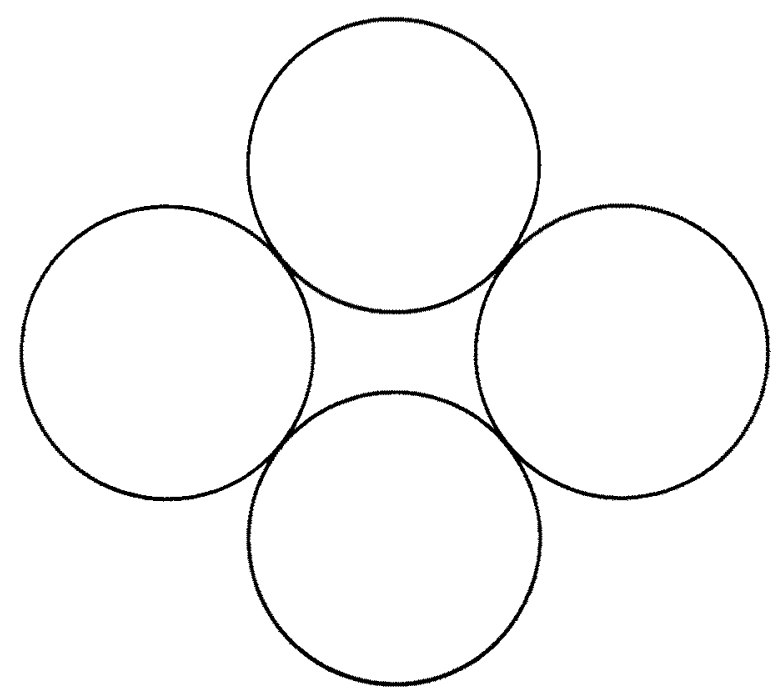

Fig. 2. Optimal packings of four pennies. The second moment is constant, as long as each penny touches its two neighbors. 
coordinates $\left\langle r_{i}, s_{i}\right\rangle$, and set $M_{r}=\sum r_{i}, M_{s}=\sum s_{i}, M_{r r}=\sum r_{i}^{2}, M_{r s}=\sum r_{i} s_{i}$, and $M_{s s}=$ $\sum s_{i}^{2}$. Then

$$
U=\left(M_{r r}+M_{r s}+M_{s s}\right)-\frac{1}{n}\left(M_{r}^{2}+M_{r} M_{s}+M_{s}^{2}\right)
$$

\section{Constructions}

We shall use three methods to construct packings.

(i) The Greedy Algorithm. A sequence of packings $\mathscr{P}_{1}, \mathscr{P}_{2}, \mathscr{P}_{3}, \ldots$ is produced by the greedy algorithm if (a) $\mathscr{P}_{1}$ contains a single point, and (b) for $n=2,3, \ldots$,

$$
\mathscr{P}_{n}=\mathscr{P}_{n-1} \cup\left\{P_{n}\right\}
$$

minimizes $U$ over all choices of $P_{n}$ satisfying (1). (There are many such sequences.)

(ii) Circular Clusters in $A_{2}$ with a Specified Center. Let $C$ be a fixed point of the plane, let $t$ be a real number, and let

$$
\mathscr{Q}=\left\{P \in A_{2}:\|P-C\| \leq t\right\}
$$

In general, the centroid of 2 will not coincide with $C$. If it does, we call 2 a circular cluster with center $C$.

The three most important cases occur when $C$ is an element of $A_{2}$, e.g.,

$$
C_{1}=\langle 0,0\rangle
$$

in oblique coordinates, $C$ is midway between two neighboring elements of $A_{2}$, e.g.,

$$
C_{2}=\left\langle\frac{1}{2}, 0\right\rangle \text {, }
$$

or $C$ is at the center of three neighboring elements of $A_{2}$, e.g.,

$$
C_{3}=\left\langle\frac{1}{3}, \frac{1}{3}\right\rangle \text {. }
$$

Figure 1(a) and (c) shows examples of clusters centered at $C_{1}$. Figure 5 below shows a cluster centered at $C_{2}$.

(iii) The Iterative Construction. The following iterative procedure produces an $n$-point packing with a small value of $U$. Choose a random initial point $T_{0}$. For $k=1,2, \ldots$ let $\mathscr{S}$ be the smallest circle around $T_{k-1}$ that contains at least $n$ points 
of $A_{2}$, let $\mathscr{R}_{k}$ be obtained by discarding just enough (randomly chosen) points from the perimeter of $\mathscr{P}$ to leave $n$ points, and let $T_{k}$ be the centroid of $\mathscr{R}_{k}$. The sequence $\mathscr{R}_{1}, \mathscr{R}_{2}, \ldots$ converges rapidly to an $n$-point circular cluster $\mathscr{R}$ in $A_{2}$.

The construction may now be repeated with a different choice for $T_{0}$, and after a number of tries the cluster with the smallest second moment is selected.

For example, when $n=16$, if the initial point $T_{0}$ is close to the origin, the algorithm usually converges in one step to the cluster shown in Fig. 1(b), which has centroid

$$
C_{16}=\left\langle\frac{1}{4}, 0\right\rangle
$$

\section{Results}

The most remarkable result, and our motivation for writing this note, is the following.

The greedy algorithm produces a unique packing for the first 75 pennies. ("Unique" means unique up to symmetry, of course. There are six ways to add a penny to Fig. 1(a), for example, but all are equivalent under symmetries of $A_{2}$.)

There are two essentially distinct choices for the 76th penny, both leading to packings with the same second moment. Whichever is chosen, the other must be chosen next, and the 77-point packing is unique (see Fig. 3). The packings now remain unique up to 313 pennies, when again there are two inequivalent choices. The 313-point packing is shown in Fig. 4, together with two inequivalent choices for the 314th penny. The two paths merge again at the 318 th penny, and the next choice occurs at the 354 th penny (see Fig. 3 ).

The pennies in Fig. 4 are labeled sequentially, so in this figure one can see the $n$-point packings produced by the greedy algorithm for all $n \leq 313$.

Beyond 354 pennies we only followed one path of the greedy algorithm, which next splits at the 426th penny.

Table 1 combines the best results produced by the three algorithms (using 100 random initial points for the iterative construction). Table 1(a) gives the smallest second moment found for all packings of $n \leq 100$ pennies. Table 1 (b) gives a selection of the best packings found for $101 \leq n \leq 500$, including all the cases where the best packing is a cluster centered at $C_{1}, C_{2}$, or $C_{3}$, and some of the

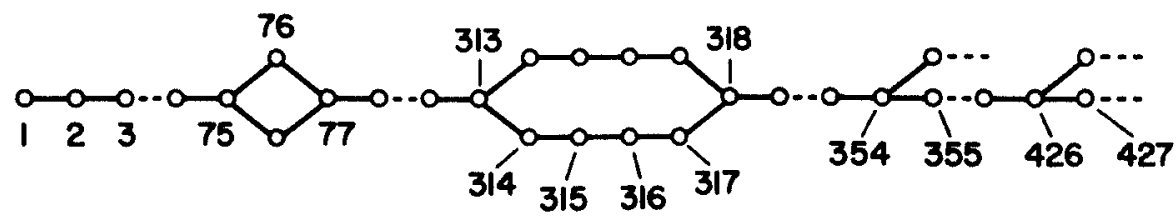

Fig. 3. Sequences of packings found by the greedy algorithm. 


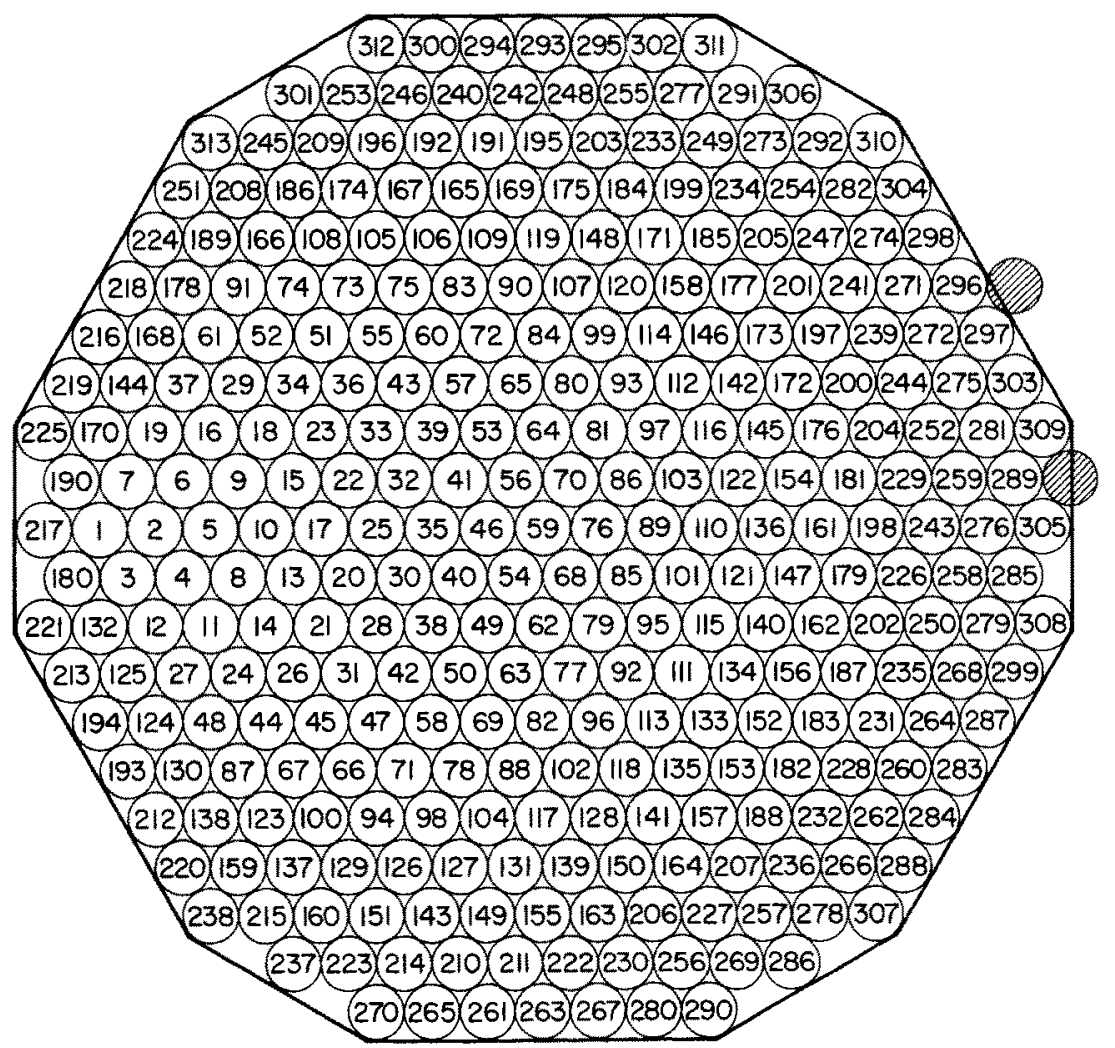

Fig. 4. Packing of the first 313 pennies found by the greedy algorithm. There are two inequivalent but eqully good choices for the 314 th penny (shaded).

cases where the greedy algorithm is the best (often just giving the beginning and end of a run of successes for the greedy algorithm).

A superscript $g$ in the table indicates that the packing is produced by the greedy algorithm, and an asterisk that it is definitely not produced by the greedy algorithm. An integer superscript $k$ denotes a circular cluster in the $A_{2}$ lattice with center $C_{k}$. We have defined $C_{1}, C_{2}, C_{3}$, and $C_{16}$ in equations (4)-(7); some other interesting centers are

$$
\begin{array}{rlrl}
C_{5} & =\left(\frac{1}{5}, \frac{1}{5}\right\rangle, & C_{6}=\left\langle\frac{1}{6}, 0\right\rangle, & C_{8}=\left\langle\frac{1}{8}, \frac{1}{8}\right\rangle, \\
C_{15}=\left\langle\frac{5}{15}, \frac{1}{15}\right\rangle, & C_{20}=\left\langle\frac{2}{20}, \frac{1}{20}\right\rangle, & C_{32}=\left\langle\frac{3}{32}, 0\right\rangle, \\
C_{44}=\left\langle\frac{1}{4}, \frac{1}{4}\right\rangle, & C_{60}=\left\langle\frac{1}{15}, 0\right\rangle, & C_{256}=\left\langle\frac{18}{256}, \frac{8}{256}\right) .
\end{array}
$$

A superscript $\alpha$ indicates that the packing is known not to be unique. (For example 48-point clusters with the same second moment may be centered at either $C_{2}$ or $C_{3}$.)

Our second discovery is that the greedy algorithm produces surprisingly good results. It finds all the best packings known for $n \leq 21$, including those of Fig. 1(a)-(c), and many clusters centered at $C_{1}, C_{2}$, or $C_{3}$ that are the best known. 


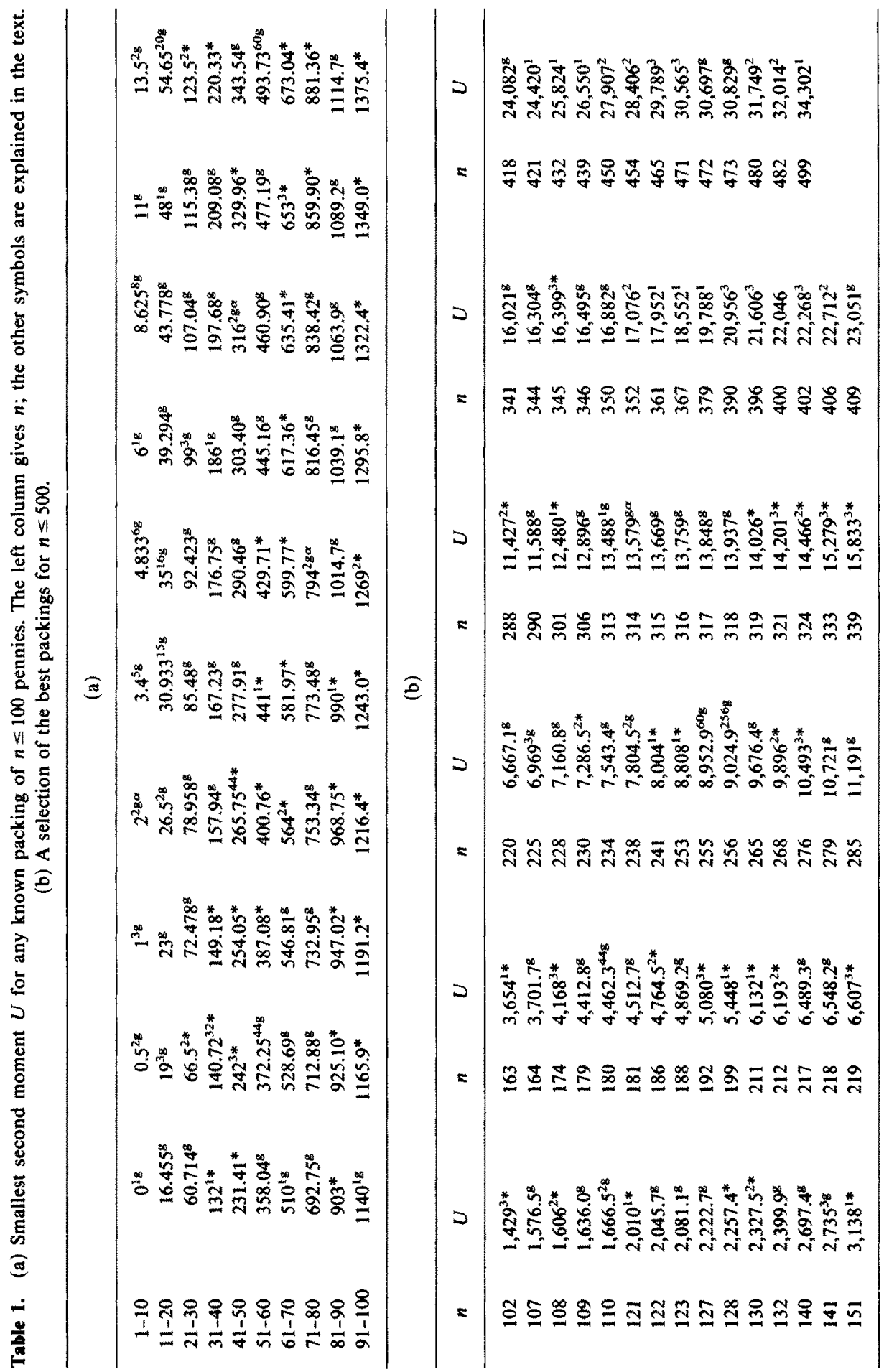


Conjecture 2. The greedy algorithm produces optimal packings for infinitely many values of $n$.

Figure 5 shows a 22-point cluster centered at $C_{2}$ that is better than that found by the greedy algorithm. The results suggest:

Conjecture 3. Circular clusters in the hexagonal lattice centered at the points $C_{1}$, $C_{2}$, or $C_{3}$ (or at equivalent points in the lattice) are optimal packings for infinitely many values of $n$. Clusters centered at any other point are optimal for only finitely many values of $n$.

For example, out of all the centers $C_{5}, \ldots, C_{256}$ defined in (7) and (8), only $C_{44}$ and $C_{60}$ occur more than once in the table.

There are also many examples where the greedy algorithm produces packings that are better than clusters centered at $C_{1}, C_{2}$, or $C_{3}$ (e.g., $n=13,6,8$, respectively).

The experimental evidence suggests that all the packings in Table 1 are optimal. Unfortunately we can only determine the optimal packings for $n \leq 5$.

Physicists have considered similar problems in three dimensions [1], [10], although not exactly from this point of view. It would be nice to know what the greedy algorithm produces in three or more dimensions.

\section{Lower Bounds on $U(n)$}

The optimal packings of one, two, and three pennies are obvious, and it is easy to show that the packings of four pennies in Fig. 2 are optimal.

Theorem 1. The five-penny packing in Fig. $6(\mathrm{a})$ is optimal, and $U(5)=3.4$.

Sketch of Proof. Let $P_{1}, \ldots, P_{5}$ be the centers of an optimal packing $\mathscr{P}$ of five pennies of diameter 1 . The corresponding adjacency graph $G$ has a node for each penny, two nodes being joined by an edge if the pennies touch. It is easy to show that the only possibilities for $G$ are the four connected graphs in Fig. $6(\mathrm{~b})-(\mathrm{e})$, in which all nodes have valency $\geq 2$. Figure $6(\mathrm{~b})$ corresponds to Fig. 6(a). The graphs in Fig. 6(c) and (d) have one degree of freedom. It is a straightforward exercise in calculus to show that the second moment $U$ for Fig. 6(c) and (d) is minimized when an extra edge occurs and the graphs reduce to Fig. 6(b).

The pentagon in Fig. 6(e) has two degrees of freedom. Let the points $\boldsymbol{P}_{\boldsymbol{i}}$ be $(-\cos \theta, 0),(0,-\sin \theta),(\cos \theta, 0),(\cos \theta-\cos \alpha, \sin \alpha)$, and $(-\cos \theta+$ $\cos \beta, \sin \beta)$. The condition $\left\|P_{4}, P_{5}\right\|=1$ implies

$$
4 \cos ^{2} \theta-4 \cos \theta(\cos \alpha+\cos \beta)+2 \cos (\alpha+\beta)+1=0 .
$$




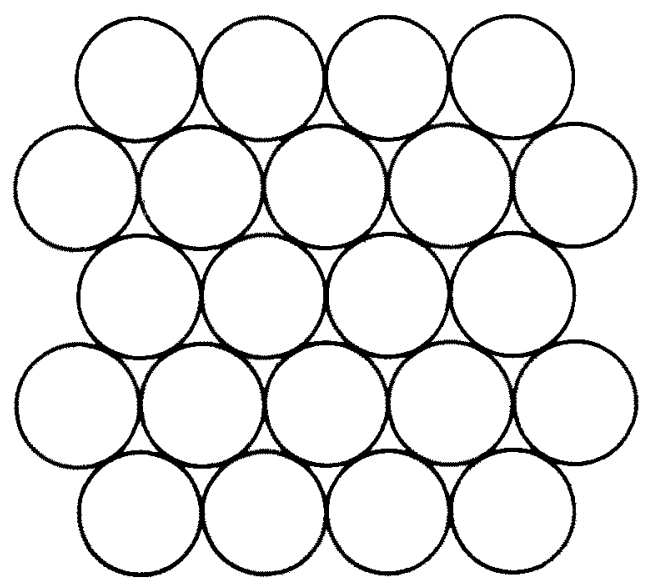

Fig. 5. A 22-point cluster centered at $C_{2}$. This is the smallest known example of a packing that is better than any found by the greedy algorithm.
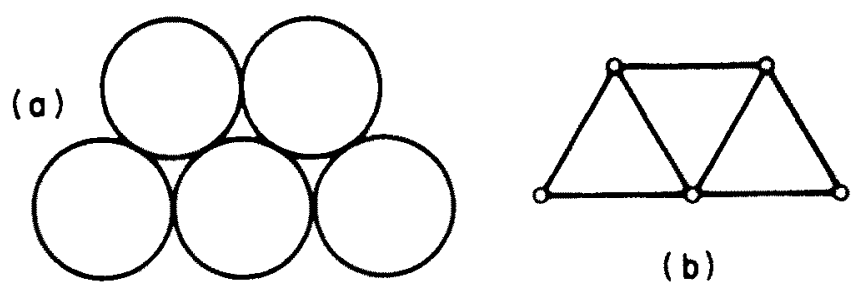

(b)

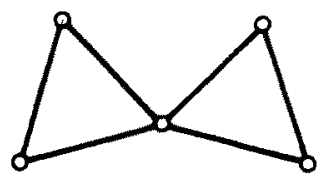

(c)

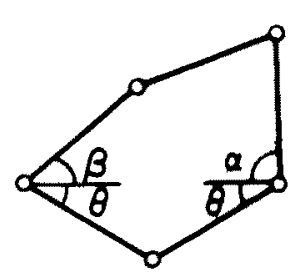

(e)

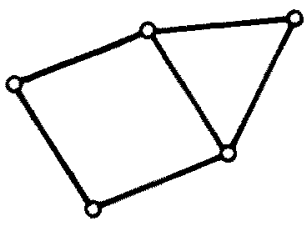

(d)

Fig. 6. (a) and (b) Optimal packing of five pennies. (c)-(e) Other arrangements of five pennies. 
Using (9) we find that

$$
\begin{aligned}
U= & \left\{\sin \theta \sin s\left(4\left(\cos ^{2} \theta+\cos ^{2} s\right)-1\right)\right. \\
& \left.+\cos \theta \cos s\left(12\left(\cos ^{2} \theta-\cos ^{2} s\right)+25\right)\right\} /(10 \cos \theta \cos s),
\end{aligned}
$$

where $s=(\alpha+\beta) / 2$. We wish to prove that there is no local minimum of $U$ in the interior of the permissible range of values of $\theta, \alpha$, and $\beta$. This will imply that the minimum of $U$ is attained at the boundary, when the graph has an additional edge and has therefore already been discussed.

The conditions $\partial U / \partial \theta=\partial U / \partial s=0$ imply

$$
\begin{gathered}
\sqrt{1-u^{2}}\left(8 v^{4}-4 v^{2}+4 u^{2}-1\right)=\sqrt{1-v^{2}}\left(24 u v^{3}\right), \\
\sqrt{1-v^{2}}\left(-8 u^{4}-4 v^{2}+4 u^{2}+1\right)=\sqrt{1-u^{2}}\left(24 u^{3} v\right),
\end{gathered}
$$

where $u=\cos s, v=\cos \theta$. We square (10) and (11) and eliminate $v$, obtaining a condition $f(u)=0$, where $f$ is a polynomial of degree 42 with integer coefficients. For each root $u$ with $-1 \leq u \leq 1$ we determine $v$ from (10) and (11) and hence $\theta, s, \alpha$, and $\beta$. It turns out that there is no solution to (10) and (11) for which the angles lie in the permissible range. This completes the proof.

Oler's inequality [6], [13] leads to a lower bound on $U(n)$ for all $n$.

\section{Theorem 2.}

$$
U(n) \geq \sum_{k=1}^{n}\left\{\left(\frac{\sqrt{3}}{2 \pi}(k-1)+\frac{3}{16}\right)^{1 / 2}-\frac{\sqrt{3}}{4}\right\}^{2}
$$

Proof. Let $\left\{P_{1}, \ldots, P_{n}\right\}$ be an optimal packing of pennies of diameter 1 , let $d_{k}=\left\|P_{k}-\bar{P}\right\|$, and assume $d_{1} \leq d_{2} \leq \cdots$. Let $C_{r}$ be a circle of radius $r$ centered at $\bar{P}$. Oler's inequality states that if

$$
k>\frac{2 \pi}{\sqrt{3}} r^{2}+\pi r+1
$$

then $P_{k} \notin C_{r}$, and so $d_{k}>r$. Therefore

$$
d_{k} \geq\left(\frac{\sqrt{3}}{2 \pi}(k-1)+\frac{3}{16}\right)^{1 / 2}-\frac{\sqrt{3}}{4}
$$

which implies (12).

Unfortunately (12) is not strong enough to imply Theorem 1, nor (for example) to show the optimality of Fig. 1(a). 
Corollary. As $n \rightarrow \infty$,

$$
U(n) \sim \frac{\sqrt{3}}{4 \pi} n^{2}
$$

Proof. It is easy to show (see [2], Theorem 4) that for a circular cluster in the hexagonal lattice centered at a lattice point we have

$$
U=\frac{\sqrt{3}}{4 \pi} n^{2}(1+o(1)),
$$

which is therefore an upper bound on $U(n)$. On the other hand (12) implies

$$
U(n) \geq \frac{\sqrt{3}}{4 \pi} n^{2}(1+o(1))
$$

which completes the proof.

\section{References}

1. C. H. Bennett, Serially deposited amorphous aggregates of hard spheres, J. Appl. Phys. 43 (1972), 2727-2734.

2. A. R. Calderbank and N. J. A. Sloane, New trellis codes based on lattices and cosets, IEEE Trans. Inform. Theory 33 (1987), 177-195.

3. C. N. Campopiano and B. G. Glazer, A coherent digital amplitude and phase modulation scheme, IEEE Trans. Comm. 10 (1962), 90-95.

4. J. H. Conway and N. J. A. Sloane, A fast encoding method for lattice codes and quantizers, IEEE Trans. Inform. Theory 29 (1983), 820-824.

5. G. Fejes Tóth, P. Gritzmann, and J. M. Wills, Finite sphere packing and sphere covering, Discrete Comput. Geom. 4 (1989), 19-40.

6. J. H. Folkman and R. L. Graham, A packing inequality for compact convex subsets of the plane, Canad. Math. Bull. 12 (1969), 745-752.

7. G. J. Foschini, R. D. Gitlin, and S. B. Weinstein, Optimization of two-dimensional signal constellations in the presence of Gaussian noise, IEEE Trans. Comm. 22 (1974), 28-38.

8. H. Groemer, Über die Einlagerung von Kreisen in einen konvexen Bereich, Math. $\mathcal{Z} .73$ (1960), 285-294.

9. M. R. Hoare, Structure and dynamics of simple microclusters, Adv. Chem. Phys. 40 (1979), 49-135.

10. M. R. Hoare and J. A. McInnes, Morphology and statistical statics of simple microclusters, $A d v$. in Phys. 32 (1983), 791-821.

11. K. Kinoshita and $\mathrm{M}$. Kobayashi, Two-dimensionally arrayed optical-fibre splicing with a $\mathrm{CO}_{2}$ laser, Appl. Optics 21 (1982), 3419-3422.

12. D. McCullough, The Great Bridge, Simon and Schuster, New York, 1972.

13. N. Oler, An inequality in the geometry of numbers, Acta Math. 105 (1961), 19-48.

14. N. J. A. Sloane and B. K. Teo, Theta series and magic numbers for close-packed spherical clusters, J. Chem. Phys. 83 (1985), 6520-6534.

15. Wegner, Über endliche Kreispackungen in der Ebene, Studia Sci. Math. Hungar., to appear.

Received December 1, 1986. 